periferia. En microscopia, el tumor tenía áreas estromales y glandulares con arquitectura en hoja, diagnosticando tumor filoide. No presentaba ningún factor de agresividad, por lo que fue considerado de bajo grado. La imunohistoquímica fue negativa para el antígeno específico de la próstata y fosfatase ácida prostática, reforzando el origen en la vesícula seminal.

Un año después de la cirugía, el paciente se encuentra bien, asintomático y sin señal de tumor residual o recidiva en los estudios de imagen.

Los tumores filoides son neoplasias con un componente epitelial y otro estromal ${ }^{1}$, que surgen frecuentemente en el seno. Raramente se desarrollan en la próstata, siendo que hay unos 15 casos publicados con origen en las vesículas seminales. Se presentan frecuentemente con síntomas del tracto urinario bajo, hematuria, hematospermia o masa al examen rectal ${ }^{1-3}$. Poseen características típicas pero no únicas en los estudios de imagen ${ }^{4}$.

El tratamiento adecuado es la exéresis quirúrgica del tumor. Estudios indican que, incluso en los menos agresivos, la recidiva local es la regla ${ }^{1}$, con algunos casos en que surgieron sarcoma ${ }^{1,5}$, invasión local y metástasis pulmonares ${ }^{1,2,6,7}$, principalmente en los de alto grado (con hipercelularidad, atipias nucleares, actividad mitótica ó aumento de la razón estroma/epitélio) ${ }^{1}$, incluso después de una cirugía radical. Como no hay metástasis linfáticas descritas, no hay indicación para linfadenectomia ${ }^{8}$.

El papel de la radioterapia y quimioterapia ${ }^{2,4,7}$ sigue por establecer, a pesar de un caso de remisión de metástasis pulmonares ${ }^{7}$.
B I B L I O G R A F Í A

1. Bostwick D, Hossain D. Phyllodes tumor of the prostate: longterm follow-up study of 23 cases. J Urol. 2004;172:894-9.

2. Fain J, Cosnow I, King B, Bostwick D. Cystosarcoma phyllodes of the seminal vesicle. Cancer. 1993;71:2055-61.

3. Schapmans S, van Leuven L, Cortvriend J, Beelaerts W, van Erps P. Phyllodes tumor of the prostate. Eur Urol. 2000;38: 649-53.

4. Olson E, Trambert M, Mattrey R. Cystosarcoma phyllodes of the prostate: MRI Findings. Abdom Imaging. 1994;19:180-1.

5. Herawi M, Epstein J. Specialized stromal tumors of the prostate: a clinicopathologic study of 50 cases. Am J Surg Pathol. 2006;30:304-694.

6. Abe $H$, Nishimura $T$, Miura $T$, Uchikoba $T$. Cystosarcoma phyllodes of the seminal vesicle. Int J Urol. 2002;9:599-601.

7. Lam $\mathrm{K}$, Yeo W. Chemotherapy induced complete remission in malignant phyllodes tumor of the prostate metastasizing to the lung. J Urol. 2002;168:1104-5.

8. Jackson D, Clements R. Sonographic and radiological features of cystosarcoma phyllodes of the Prostate. Clinical Radiology. 1999;54:473-85.

R.O. Soares*, T.P. Correia, A. Cardoso y M. Cerqueira

Servicio de Urología, Hospital Pedro Hispano, Matosinhos, Portugal

Autor para correspondencia.

Correo electrónico: ricardosoares81@gmail.com (R.O. Soares).

\title{
Pseudoaneurisma de arteria renal tras nefrectomía parcial laparoscópica. Diagnóstico, tratamiento y revisión bibliográfica
}

\section{Renal artery pseudoaneurysm after partial nephrectomy. Diagnosis, treatment and literature review}

\section{Sr. Director:}

Desde mayo de 2006 hasta diciembre de 2008 se han realizado 9 nefrectomías parciales laparoscópicas (NPL) en nuestro servicio. Presentamos el caso de un varón de 61 años diagnosticado de forma incidental de un tumor renal derecho de $2,5 \mathrm{~cm}$, en polo inferior.

Se practicó NPL con un tiempo quirúrgico de $220 \mathrm{~min}$, un tiempo de isquemia de $32 \mathrm{~min}$ y un sangrado de $200 \mathrm{ml}$. La resección se realizó con corte frío y un margen de seguridad de $1 \mathrm{~cm}$. Posteriormente, se suturó la vía urinaria y el lecho quirúrgico con poliglactina $3-0$, sobre dicho lecho de resección se añadió Floseal $^{\circledR}$ y se colocó una membrana de Surgicel ${ }^{\circledR}$. La sutura del parénquima se realizó mediante poliglactina del 1 y Hemo-locks ${ }^{\circledR}$ para mantener tensión.
El resultado anatomopatológico fue carcinoma de células claras con áreas de crecimiento papilar, grado II de Furhman. El tamaño tumoral fue de $2,7 \mathrm{~cm}$, con márgenes quirúrgicos, cápsula y grasa perirrenal libres de infiltración.

Finalizada la cirugía presentó hematuria leve, que $24 \mathrm{~h}$ después coincidiendo con el inicio de la deambulación se agravó requiriendo lavado continuo y transfusión de 2 concentrados de hematíes. El paciente fue dado de alta sin sonda a los 4 días de la intervención.

Cuatro días después del alta, el paciente presentó episodio de hematuria y pérdida de conciencia. Se realizó una TC (fig. 1), observando en el riñón derecho una imagen sacular de $2,5 \mathrm{~cm}$ que capta contraste en fase arterial y no en fase excretora; sospechando la existencia de un pseudoaneurisma de arteria renal (PAR). 
a

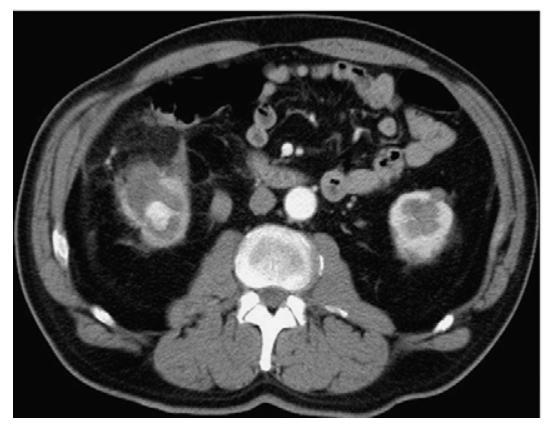

b

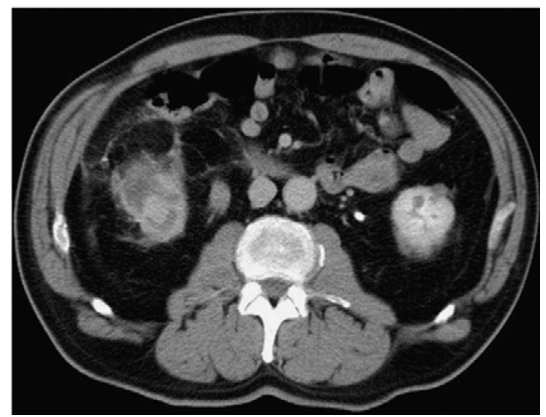

Figura 1 - TC realizado tras la aparición de hematuria en el paciente intervenido de NPL, donde se observa una colección de $2,5 \mathrm{~cm}$ en riñón derecho que: A) capta contraste en fase arterial, B) y no en fase excretora.

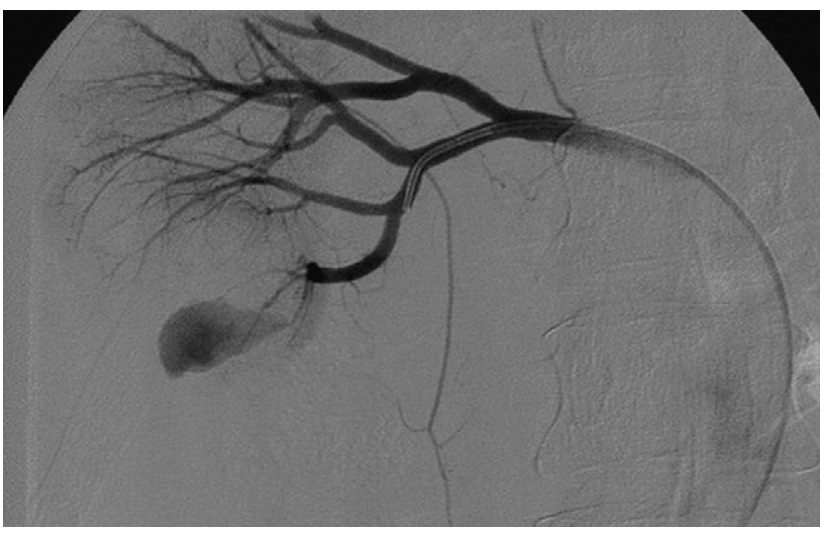

Figura 2 - Arteriografía de arterial renal derecha mostrando a nivel de la $3 .^{a}$ bifurcación, una imagen sacular correspondiente al PAR.

Se practicó arteriografía observando la fuga de contraste a nivel de la 3 . $^{\text {a }}$ bifurcación de la arteria renal, originando una colección que comunicaba con la vía urinaria. Se procedió a la embolización selectiva mediante coils (fig. 2), resolviéndose la fístula.

El PAR es una complicación rara, descrita tras procedimientos renales percutáneos (biopsia renal, nefrostomía, y nefroureterolitotomía percutánea), traumatismos renales, litotricia ureteroscópica, incluso tras trasplante renal.

Es poco frecuente tras una nefrectomía parcial abierta, con una incidencia del $0,43-7,9 \%^{1,2}$. Tras una NPL, hasta la actualidad, existen 21 casos publicados de PAR, recogidos y revisados en la tabla 1 . Singh-Gill ${ }^{3}$ reportan una incidencia de PAR tras NPL del 1,7\%.

Algunos autores plantean que tras una nefrectomía parcial abierta los vasos seccionados se identifican y son suturados más fácilmente, que con la técnica laparoscópica ${ }^{4}$.

Existen teorías que pretenden explicar la etiología del $\mathrm{PAR}^{3}$ :

- Intraoperatoriamente no se evidencia sangrado de la arteria seccionada por: ser ligada parcialmente, TA baja o espasmo arterial ${ }^{5}$. Cuando el paciente incrementa su actividad, el trombo que obstruía la arteria se deshace, produciéndose el sangrado.
- Sutura de aproximación del parénquima renal subóptima, sin suficiente tensión.

- Reabsorción de la sutura aplicada al lecho tumoral ${ }^{6}$, correspondiendo el tiempo hasta la aparición del pseudoaneurisma con el tiempo de reabsorción de la sutura.

La arteria sangrante vierte a una cavidad cerrada situada en el lecho de resección, constituyendo el pseudoaneurisma. La hematuria asociada sería debida a la apertura del pseudoaneurisma a la vía urinaria ${ }^{2}$, que suele ocurrir entre la $2 .^{a}-4 .^{a}$ semana postoperatoria.

$\mathrm{Nadu}^{7}$ observa una incidencia de PAR del 7,5\% para tumores centrales (invaden en profundidad el parénquima renal llegando a estar en contacto o invadiendo el sistema colector y/o el seno renal), y del $0 \%$ para periféricos, postula que las NPL de tumores centrales deberían ser realizadas en centros con servicio de radiología intervencionista. Sin embargo, en nuestra revisión, la mayoría de los PAR aparecen en NPL de tumores periféricos.

Existen diferentes técnicas para mejorar la hemostasia y prevenir la formación de PAR tras la NPL:

- Suturar el lecho quirúrgico de forma minuciosa ${ }^{5,8}$.

- Aplicar sobre lecho quirúrgico agentes que favorezcan la hemostasia (Floseal ${ }^{\circledR}$ y/o Surgicel ${ }^{\circledR}$ ) siendo complementarios a una correcta sutura hemostática ${ }^{5,6}$.

- Mejorar la sutura del lecho usando clips Lapra-Ty o bien un sistema de tracción de la sutura ${ }^{5}$.

- Desclampar el pedículo tras la $1 .^{a}$ sutura del lecho de resección para identificar los vasos no suturados causantes del sangrado 4 .

- Hiperinsuflación pulmonar tras desclampar el pedículo, para incrementar la presión venosa central y detectar mejor la presencia de sangrado ${ }^{5}$.

De los 21 casos publicados de PAR tras NPL, el tamaño tumoral medio fue de 2,8 cm (1,5-5), 19 eran CCR y 2 AML, 12 eran derechos y 6 izquierdos. El tiempo medio de isquemia fue de 37,6 min (23-68). La clínica inicial fue hematuria $77 \%$, dolor en flanco $41 \%$, y menos frecuentemente debilidad, disnea, hipotensión ortostática, mareo o síncope. La media de días hasta la aparición de hematuria tras la NPL fue de 19,7 (1-90). 
Tabla 1 - Revisión bibliográfica de todos los casos publicados en la literatura internacional

\begin{tabular}{|c|c|c|c|c|c|c|c|c|c|c|c|c|c|c|c|c|}
\hline Artículo & Edad & Sexo & AP & $\begin{array}{l}\text { Tamaño } \\
\text { tumor }\end{array}$ & Lugar & Lado & $\begin{array}{l}\text { Tiempo } \\
\text { Isquemia }\end{array}$ & Sutura & Margen & Días & Clínica & Diagnóstico & $\begin{array}{l}\text { Orden } \\
\text { Arteria }\end{array}$ & $\begin{array}{c}\text { Tamaño } \\
\text { PAR }\end{array}$ & $\begin{array}{c}{[]} \\
\text { Hem }\end{array}$ & Tto \\
\hline \multirow[t]{2}{*}{ Moore et al } & 53 & M & $\begin{array}{l}\text { CCR } \\
\text { Pap. }\end{array}$ & ND & Perif & I & ND & ND & ND & ND & Hematuria & TC & ND & $\approx 4$ & ND & Coils \\
\hline & 60 & M & $\begin{array}{l}\mathrm{CCR} \\
\text { Claras }\end{array}$ & ND & Perif & D & ND & ND & ND & ND & Hematuria & TC & ND & 2 & ND & Coils \\
\hline \multirow[t]{7}{*}{$\operatorname{Singh}^{3}$} & $\begin{array}{r}59 \\
(34- \\
80)\end{array}$ & M & $\mathrm{CCR}$ & 2,6 & $\begin{array}{l}\text { Invasión } \\
\text { Media 1,8 }\end{array}$ & D & 31 & $\begin{array}{c}\text { L: 2-0 } \\
\text { Polyglactin+ } \\
\text { P: O Polyglactin } \\
\text { sobre celulosa. } \\
\text { Floseal }^{\mathbb{1}} \text { en } 2 \\
\text { casos }^{\text {coso }}\end{array}$ & Neg & 11 & Hematuria & Angiografía & 3. & ND & 7 & Coils \\
\hline & " & M & $\mathrm{CCR}$ & 5 & " & D & 31 & “ & Neg & 15 & $\begin{array}{c}\text { Dolor abd } \\
\text { Debilidad } \\
\text { Dificultad resp }\end{array}$ & Angiografía & 3. & ND & 9 & Coils \\
\hline & " & м & $\mathrm{CCR}$ & 2,2 & " & D & 33 & " & $\mathrm{Neg}$ & 8 & Sangrado peri JP & Angiografía & 4. & ND & 3 & Coils \\
\hline & “ & $\mathrm{F}$ & $\mathrm{CCR}$ & 3 & " & D & 30 & " & $\mathrm{Neg}$ & 14 & Dolor flanco & Angiografía & 3. & ND & ND & Coils \\
\hline & " & $\mathrm{F}$ & CCR & 4 & " & D & 45 & " & $\mathrm{Neg}$ & 12 & Hematuria & Angiografía & 4. & $\mathrm{ND}$ & 6 & Coils \\
\hline & " & $\mathrm{F}$ & AML & 4,6 & $"$ & D & 39 & " & $\mathrm{Neg}$ & 10 & $\begin{array}{l}\text { Hipotensión } \\
\text { ortostática }\end{array}$ & Angiografía & 3. ${ }^{\circ}$ & ND & 5 & Coils \\
\hline & 63 & M & CCR & 1.5 & Perifa & $\mathrm{I}$ & 45 & L. $2-0$ & $\mathrm{Neg}$ & $9 y$ & $\begin{array}{c}\text { Dolor en flanco } \\
\text { Hematuria }\end{array}$ & TC & Segmentaria & 3 & ND & Coils \\
\hline \multirow{2}{*}{ Wright $^{4}$} & & & $\begin{array}{l}\text { Pap: } \\
\text { pT1 } \\
\text { G2/4 }\end{array}$ & & (Sup) & & & $\begin{array}{l}\text { Glycolide/ } \\
\text { lactide } \\
\text { P: } 0 \text { Glycolide/ } \\
\text { lactide sobre } \\
\text { celulosa }\end{array}$ & & 11 & Dolor flanco & & & & & \\
\hline & 59 & $\mathrm{~F}$ & $\begin{array}{l}\text { CCR } \\
\text { C1.: } \\
\text { pT1 } \\
\text { G2/4 }\end{array}$ & 1,6 & $\begin{array}{l}\text { Perif } \\
\text { (Sup) }\end{array}$ & D & 23 & $\begin{array}{l}0 \text { Glycolide/ } \\
\text { lactide sobre } \\
\text { celulosa } \\
\text { FloSeal }^{\mathbb{\mathbb { E }}}\end{array}$ & Neg & 9 & $\begin{array}{c}\text { Fatiga, Diarrea } \\
\text { Dolor flanco } \\
\text { Microhematuria }\end{array}$ & TC & $\begin{array}{c}\text { Sub- } \\
\text { segmentaria }\end{array}$ & ND & ND & Coils \\
\hline Negoro $8, a$ & 36 & $\mathrm{~F}$ & $\begin{array}{l}\text { CCR } \\
\text { Cl.: } \\
\text { pT1a } \\
\text { G2/4 }\end{array}$ & 3,3 & $\begin{array}{l}\text { Perif } \\
\text { (Med) }\end{array}$ & D & $68^{\mathrm{b}}$ & $\begin{array}{l}\text { L: 3-0 Vicryl } \\
\text { P: 2-0 } \\
\text { Polysorb }\end{array}$ & ND & ND & Hematuria y RAO & $\begin{array}{l}\text { TC; ECO } \\
\text { doppler }\end{array}$ & $\begin{array}{l}\text { Segmentaria } \\
\text { Media }\end{array}$ & 3 & 0 & Coils \\
\hline Hayn et al & 58 & M & $\begin{array}{l}\text { CCR } \\
\text { Cl.: } \\
\text { pT1a } \\
\text { G3/4 }\end{array}$ & 2 & Perif (Inf) & I & ND & ND & Neg & 90 & $\begin{array}{l}\text { Hematuria } \\
\text { Dolor flanco } \\
\text { Mareo Disnea }\end{array}$ & $\begin{array}{c}\text { TC; ECO } \\
\text { doppler; } \\
\text { Arteriografía }\end{array}$ & ND & $\approx 1 \mathrm{y} \approx 3$ & 0 & Coils \\
\hline Zorn $^{5}$ & 49 & $\mathrm{~F}$ & AML & 2,5 & $\begin{array}{l}\text { Perif } \\
\text { (Post) }\end{array}$ & D & 40 & $\begin{array}{l}\text { Coag con } \\
\text { Argón. } \\
\text { L: 4-0 Vicryl } \\
\text { FloSeal } \\
\text { P: 2-0 Vicryl } \\
\text { LapraTy para } \\
\text { Ly P }\end{array}$ & Neg & 24 & $\begin{array}{l}\text { Dolor en flanco } \\
\text { Hematuria }\end{array}$ & TC & 3. & 1,6 & $>1$ & Coils \\
\hline \multirow[t]{2}{*}{ Uberoi $^{6}$} & 41 & M & $\begin{array}{l}\text { CCR } \\
\text { Pap }\end{array}$ & 1,5 & $\begin{array}{c}\text { Perif } \\
\text { (Anterior- } \\
\text { central) }\end{array}$ & & & & & & & & & & & Coils \\
\hline & 56 & M & $\begin{array}{l}\mathrm{CCR} \\
\mathrm{Cl} .\end{array}$ & 1,5 & $\begin{array}{l}\text { Perif (Inf) } \\
\text { Invade } \\
1 \mathrm{~cm}\end{array}$ & D & $33^{\mathrm{b}}$ & $\begin{array}{l}\text { L: Polyglactin } \\
\text { 2-0+Surgicel }\end{array}$ & Neg & 21 & Hematuria & $\begin{array}{l}\text { Angio-RM } \\
\text { Arteriografía+ } \\
\text { corticoides }\end{array}$ & $\begin{array}{l}\text { Segmentaria } \\
\text { Inferior }\end{array}$ & 3 & ND & Coils \\
\hline $\begin{array}{l}\text { Cohenpour } \\
\text { et al }\end{array}$ & 68 & M & $\mathrm{CCR}$ & $\mathrm{ND}$ & $\mathrm{ND}$ & I & ND & $\begin{array}{c}\text { P: Polyglactin } 0 \\
\text { ND }\end{array}$ & ND & 14 & Hematuria & TC & ND & 2,5 & ND & Coils \\
\hline $\begin{array}{l}\text { Shigeta } \\
\text { et al }\end{array}$ & 57 & M & $\begin{array}{l}\text { CCR } \\
\text { Pap: }\end{array}$ & 2,7 & $\begin{array}{c}\text { Perif } \\
\text { (Med-Inf) }\end{array}$ & I & $49^{\mathrm{b}}$ & $\begin{array}{l}\text { L: Polyglactin } \\
3-0\end{array}$ & & 42 & Dolor flanco & TC & $\mathrm{ND}$ & ND & ND & Coils \\
\hline
\end{tabular}




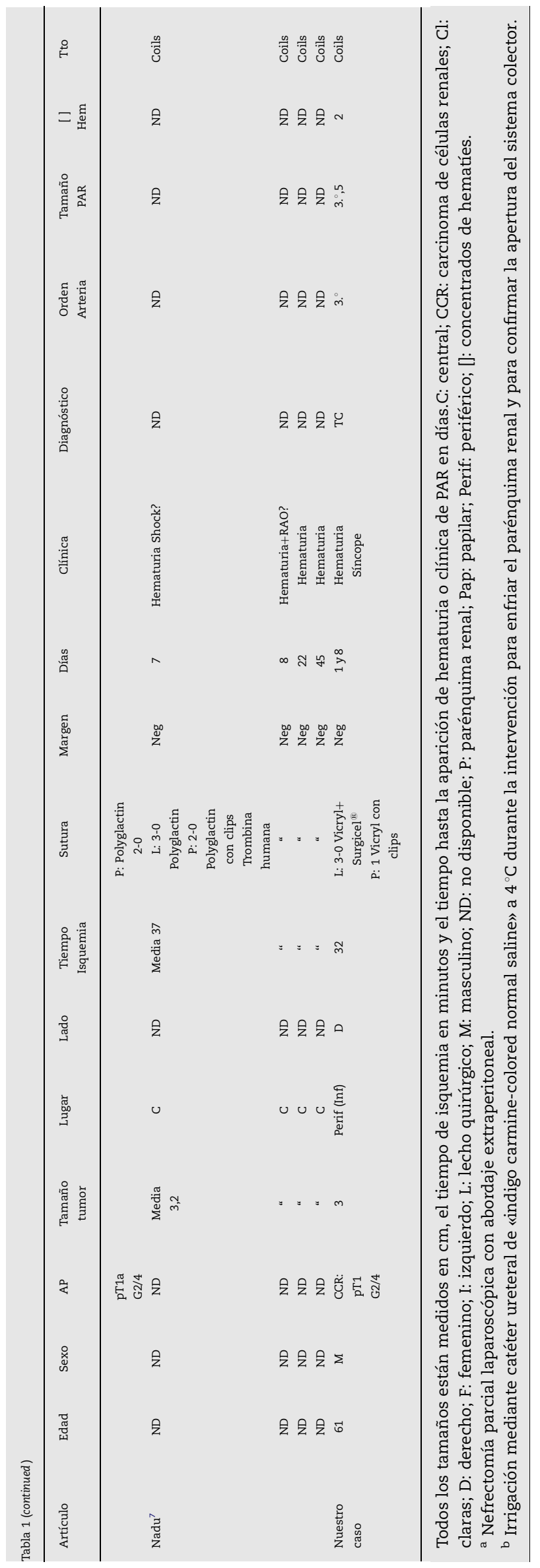

El diagnóstico de PAR exige la sospecha clínica tras la presencia de hematuria con o sin dolor en flanco entre la 2. ${ }^{a}$ y 4. ${ }^{\text {a }}$ semana tras la NPL, debiendo realizar TC con contraste en fase arterial, venosa y excretora. Es la técnica diagnóstica de elección, realizada en el $61 \%$ de los casos publicados; la arteriografía se realizó en el 44,4\% y en un paciente alérgico a contraste se realizó una angio-RM. La TC muestra habitualmente una imagen sacular, en la zona correspondiente al lecho quirúrgico, que capta contraste en fase arterial y no en fase venosa, ni excretora.

Puede ser difícil distinguir un PAR de un urinoma en la $\mathrm{TC}^{9}$, de ahí la importancia de realizar la TC en distintas fases y una arteriografía de arteria renal, en la cual podremos observar la fuga de contraste, generalmente a nivel de la $3 .^{a}-4 .^{\text {a }}$ dando una imagen sacular de tamaño similar a la del TC.

El tratamiento de elección del PAR en un paciente estable hemodinámicamente es la arteriografía con embolización selectiva mediante coils de la arteria sangrante, produciéndose una mínima pérdida de parénquima renal ${ }^{10}$, es una técnica con tasa de complicaciones baja comparada con las maniobras quirúrgicas, y presenta un índice de éxito mayor del $80 \%^{8}$. En todos los casos revisados fue el tratamiento de elección y definitivo. Se recomienda la realización de pruebas de imagen que confirmen la resolución del pseudoaneurisma ${ }^{4}$.

B I B L I O G R A F Í A

1. Van Poppel H, Kilen K, Baert L. Incidental renal cell carcinoma and nephron sparing surgery. Curr Opin Urol. 2001;11:281.

2. Albani JM, Novick AC. Renal artery pseudoaneurysm after partial nephrectomy: three case reports and a literature review. Urology. 2003;62:227.

3. Singh D, Gill I. Renal artery pseudoaneurysm following laparoscopic partial nephrectomy. J Urol. 2005;174:2256-9.

4. Wright JL, Porter JR. Renal artery pseudoaneurysm after laparoscopic partial nephrectomy. Urology. 2005;66:1109.e17-9.

5. Zorn KC, Starks CL, Ofer MD, Orvieto MA, Shalhav AL. Embolization of Renal-Artery Pseudoaneurysm after laparoscopic partial nephrectomy for angiomyolipoma: case report and literature review. J Endourol. 2007;21:763-8.

6. Uberoi J, Badwan KH, Wang DS. Renal-Artery pseudoaneurysm after laparoscopic partial nephrectomy. J Endourol. 2007;21:330-3.

7. Nadu A, Kleinmann N, Laufer M, Dotan Z, Winkler H, Ramon J. Laparoscopic partial nephrectomy for central tumors: Analysis of perioperative outcomes and acomplications. J Urol. 2009;181:42-7.

8. Negoro H, Kawakita M, Koda Y. Renal artery pseudoaneurysm after laparoscopic partial nephrectomy for renal cell carcinoma in a solitary kidney. Int J Urol. 2005;12:683-5.

9. Moore CJ, Rozen SM, Fishman EK. Two cases of pseudoaneurysm of the renal artery following laparoscopic partial nephrectomy for renal cell carcinoma: CT angiographic evaluation. Emerg Radiol. 2004;10:193-6.

10. Chatziioannou A, Brountzos E, Primetis E, Malagari K, Sofocleous C, Mourikis D, et al. Effects of superselective embolization for renal vascular injuries on renal parenchyma and function. Eur J Vasc Endovasc Surg. 2004;28: 201-6. 
I.J. Povo-Martín ${ }^{\mathrm{a}, *}$, D. Gallego-Vilar ${ }^{\mathrm{a}}$, M. Rodrigo-Aliaga ${ }^{\mathrm{a}}$ y J. García-Vilab

aservicio de Urología, Hospital General de Castellón, Castellón, España
bServicio de Radiología, Hospital General de Castellón, Castellón, España

*Autor para correspondencia.

Correo electrónico: Ivanjp82@hotmail.com (I.J. Povo-Martín).

\section{Melanoma de uretra masculina: caso clínico}

\section{Melanoma of male urethra: A clinical case}

\section{Sr. Director:}

Presentamos el caso de un varón de 71 años que manifestaba uretrorragia de diez días de evolución y aparición de una lesión pigmentada a través del meato urinario, sin otra clínica asociada. La exploración física, incluyendo la palpación de cadenas linfáticas inguinales, así como el hemograma y la bioquímica sanguínea, incluyendo marcadores tumorales, eran rigurosamente normales. La cistoscopia reveló la presencia de una lesión nodular, friable y grisácea, de unos $17 \times 7 \mathrm{~mm}$, en la fosa navicularis, que fue biopsiada. La anatomía patológica demostró una proliferación de células poligonales, con núcleos esféricos e hipercromáticos, que se teñían positivamente para HMB45, melanina A, vimentina y S100, siendo negativa para queratina. Todo ello fue compatible con el diagnóstico de melanoma primario de la uretra. Una TAC toracoabdominopélvica descartó afectación metastásica ganglionar o visceral. En ese punto, se realizó amputación parcial del pene, cuyos márgenes quirúrgicos fueron negativos. Se realizó técnica del ganglio centinela inguinal bilateral, resultando negativo, por lo que no se realizó linfadenectomía. Los resultados de la biopsia de uretra fueron confirmados en la pieza quirúrgica.

Durante el seguimiento de este paciente, se realizó una PET/ TAC a los seis meses y al año de la cirugía, que fueron normales. Seis meses después, el paciente presentó dolor y distensión abdominal de dos semanas de evolución, con signos de ascitis en la exploración, lo que llevó a la realización de una TAC toracoabdominal, que confirmó la presencia de líquido ascítico, así como engrosamiento del peritoneo, mesenterio, un conglomerado adenopático retroperitoneal, metástasis hepáticas bilobares de hasta $6 \mathrm{~cm}$, nódulos pulmonares múltiples y derrame pleural bilateral (fig. 1). En la analítica de sangre destacaba una LDH de $833 \mathrm{UI} /$. Se realizó una paracentesis, con obtención de líquido serohemático. Con el diagnóstico de recurrencia metastásica de melanoma, se inició tratamiento con quimioterapia paliativa con dacarbazina intravenosa, a dosis de $250 \mathrm{mg} / \mathrm{m}^{2}$ durante cinco días. Tres semanas después, el paciente presentó ascitis hemorrágica y fallo multiorgánico, añadiéndose pancitopenia secundaria al tratamiento con quimioterapia paliativa con dacarbazina intravenosa, lo que ocasionó la muerte del paciente pocos días después, a pesar del tratamiento farmacológico y de soporte.
Con respecto al melanoma de uretra, cabe destacar que es una variante muy infrecuente de melanoma, más aun en el varón, que conlleva un pronóstico infausto dada la naturaleza agresiva de la enfermedad y por su diagnóstico generalmente en estadios avanzados. Dada la dificultad de acceso de estas lesiones, los procesos de prevención secundaria de este tumor no son rentables. Aproximadamente un tercio de los pacientes tiene afectación linfática al diagnóstico, con una supervivencia global a los cinco años inferior al $30 \%{ }^{1,2}$. La clasificación de Breslow en estos melanomas es insuficiente, dada la mayor agresividad de esta enfermedad respecto a los melanomas cutáneos, por lo que en la práctica clínica se clasifican en: estadio i-II para la enfermedad localizada; estadio III si hay afectación linfática regional (inguinal), y estadio iv para la enfermedad metastásica ${ }^{2}$. Con respecto al tratamiento, sólo los estadios I-III son potencialmente curables, mediante cirugía lo más agresiva posible (penectomía total o parcial, con linfadenectomía si hay afectación ganglionar), con márgenes quirúrgicos microscópicos de al menos $2 \mathrm{~cm}$ libres de enfermedad, puesto que la uretrectomía parcial está asociada con un riesgo de recurrencia local del $50-70 \%$ en el primer año ${ }^{3}$. Sin embargo, este objetivo es difícil de alcanzar en los melanomas de uretra masculina, dado lo traumático de la amputación. Las técnicas de exenteración se reservan para pacientes con objetivo paliativo ${ }^{4}$.

La inmunoterapia adyuvante está indicada en casos con enfermedad residual tras la cirugía o en aquellos en los que

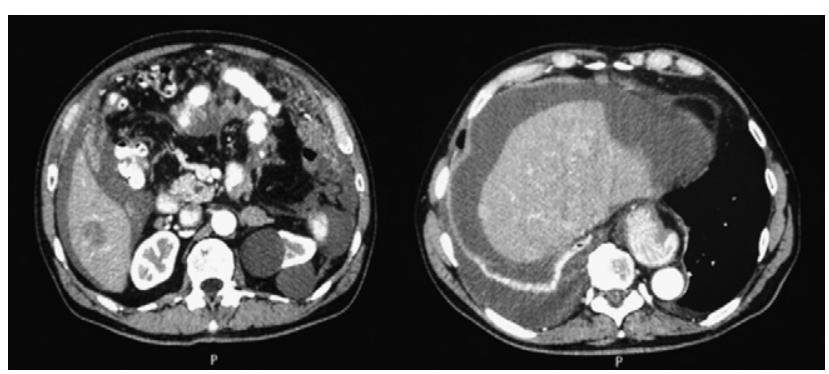

Figura 1 - Imágenes de la tomografía axial computarizada abdominal, en la que se objetivan metástasis hepáticas, líquido ascítico y nódulos retroperitoneales. 